\title{
Algunas NOTAS SOBRE ARQUEOLOGÍA Y COLONIALISMO. LA ZONA ESPAÑOLA DEL PROTECTORADO MARROQUÍ, 1912-1945
}

\author{
Enrique Gozalbes Cravioto \\ Universidad de Castilla-La Mancha \\ Manuel J. Parodi Álvarez \\ Universidad de Cádiz \\ JaVier Verdugo SANTOS \\ Consejería de Cultura, Junta de Andalucía
}

Recibido: 16/11/2012

Revisado: 22/11/2012
Aceptado: 26/11/2012

Publicado: 17/06/2013
RESUMEN

A lo largo de la primera mitad del siglo XX se desarrollaron en el Norte de Marruecos y en el seno de la administración conjunta hispano-marroquí del Protectorado ejercido por España sobre dichos territorios las estructuras de gestión del Patrimonio Arqueológico de dicha Zona. En los párrafos de este artículo abordaremos el paulatino proceso de construcción de dichas estructuras, que marchó en paralelo al desarrollo de los trabajos de investigación de campo y, en buena medida, de forma subsiguiente a los mismos.

\section{Palabras Clave}

España, Marruecos, Protectorado, Arqueología, Patrimonio, Investigación.

\section{ABstRact}

Through the several decades belonging to the first half of the $\mathrm{XX}^{\text {th }}$ Century, the territories of North Marocco, placed under the Spanish Protectorate since the Conference of Algeciras in 1906, experienced the development of real public structures devoted to the management of that area's Archaeological Heritage. We shall focus on this phenomenon, which is closely related to the development of archaeological research in itself as well.

\section{KEYWORDS}

Spain, Morocco, Protectorate, Archaeology, Heritage, Research. 
Marruecos habría de constituir uno de los últimos capítulos del reparto colonial del Norte de África (junto a Libia y el especial caso egipcio). Pese a su situación permanente de crisis, causada por la debilidad de su Estado, el Imperio Cherifiano arrastraba una larguísima tradición, varias veces centenaria, de organización nacional, por lo que no podía asimilarse sin más como simple colonia. Este hecho histórico, más mal que bien, también había influido de alguna manera en la arqueología, si bien de forma bastante marginal. Aunque la organización de la arqueología, como todos los sistemas de un país moderno, fue implantada por la administración del Protectorado, por los franceses y los españoles en sus respectivas zonas, lo cierto es que alguna muy débil tradición existía al respecto en el país.

No vamos a remontarnos en el tiempo, por ejemplo a los permisos concedidos a diferentes viajeros en el siglo XVIII para la visita de restos romanos en Volubilis (Euzennat, 1956). El inicio de la arqueología de campo, más allá de las percepciones de los viajeros y datos sueltos de diplomáticos, se produjo con las actuaciones del cónsul francés Charles Tissot, un personaje muy importante (era también doctor en Historia) que previamente había realizado una relevante labor arqueológica en Túnez. Entre 1870 y 1874 el gobierno del Sultán protegió al diplomático francés Charles Tissot, dotándolo además de una guardia para su seguro, en los distintos recorridos en busca de restos romanos (Rebuffat, 2000).

En la misma época, por el contrario, otro diplomático, el español Teodoro de Cuevas, en sus recorridos arqueológicos y geográficos por el Norte del país, donde también localizó bastantes vestigios antiguos, tenía que contratar en cada caso una guardia armada, y se veía muchas veces ante el peligro (Gozalbes, 2005; 2008a). Ello imposibilitaba la realización de los estudios, más allá de Tánger, puerto de entrada en el país, que era abierto y seguro para los europeos.

Década y media más adelante otro diplomático francés, Henry de la Martinière, siguió la estela de Charles Tissot, pero como el grado de seguridad había mejorado algo, pudo desviarse algo más (no mucho) de la ruta principal. En sus memorias sobre Marruecos, Martinière recordaba que en los desplazamientos por el país precisaba de llevar una carta del Sultán que era un pasaporte para los "pachas" o gobernadores, ordenando se le atendiera y diera facilidades para su misión, pero aclaraba que ese do- cumento, precioso en unas zonas, era absolutamente inútil donde se hallaban "tribus insumisas". Lo principal fue que terminó por conseguir del Sultán una orden escrita (un Firman), el único de su género, que autorizaba la realización de excavaciones arqueológicas, y facilitaba la contratación de trabajadores y la seguridad de los trabajos. Dichas excavaciones oficiales se realizaron en 1891 y 1892 en Volubilis y en Lixus (Martinière, 1919, 302 y ss.).

En los primeros años del siglo XX el Gobierno francés, previendo la futura colonización de Marruecos, decidió crear la Mision Scientifique du Maroc, con centro en la ciudad de Tánger. Primero pondrá al frente de la misma al geólogo Gaston Buchet, que mostrará muchísimo interés por la arqueología, y más tarde (a partir de 1906) al sociólogo Edouard-Leon Michaux-Bellaire. La Misión realizará una gran labor en el terreno de la arqueología, con la metodología propia de la época, excavará en las grutas de Achacar (Neolítico y Prehistoria Reciente), en necrópolis de cistas de la Edad del Bronce (sobre todo en El Mries), así como en tumbas púnicas (Magoga Srira), de época mauritana (necrópolis de Marshan), así como en la necrópolis romana de Tánger, con resultados espectaculares para aquella época, y que dieron origen a las publicaciones científicas de arqueología de $\mathrm{Ma}-$ rruecos (Gozalbes, 2008b).

En el resto del país, la descomposición del Estado, producida por implosión pero sobre todo derivada de las intervenciones europeas, alejaba de toda preocupación por los restos arqueológicos. Es obvio que la mentalidad estaba muy alejada de la atención, que seguía anclada naturalmente en la visión de los "tesoros". Antigüedades que salían al mercado en Tánger o Larache, no digamos ya los millares de monedas antiguas (en Tánger en los siglos XIX y XX muchísimos europeos y norteamericanos formaron colecciones), salían irremisiblemente del país. En Volubilis y en Lixus había hornos de cal que aprovechaban los mármoles antiguos.

En todo caso, en estos últimos años el Sultán promulgó una orden en la que prohibía la realización de excavaciones y de búsquedas arqueológicas en el Imperio Cherifiano. Fuera de la excepción de Tánger, ello impidió la realización de estudios, problemáticos por las condiciones, pero no la actuación de buscadores clandestinos. En estos momentos salieron al mercado dos tesorillos de monedas, de características bien diferentes. El primero hacia 1906, de moneda cartaginesa de la época de la Se- 
gunda Guerra Púnica, conocido como “Tesoro de Tánger”, y que tan sólo sería publicado en 1999 por Leandre Villaronga. El segundo muy poco tiempo más tarde, un tesoro de varios miles de monedas del rey Juba II, de los primeros años de la era cristiana, y que aunque se difundiera como "tesoro de Alcazar(quivir)", hoy sabemos que el hallazgo se produjo en los alrededores de Banasa.

\section{LOS PRIMEROS PASOS: 1912-1939}

En 1912 se establece el Protectorado en Marruecos. Se trataba inicialmente de un Protectorado francés, aunque a España se le garantizaba una "zona de influencia" en el Norte, entre las ciudades españolas de Ceuta y Melilla, rápidamente convertida por los españoles de hecho en "Zona de Protectorado de España”. El hecho iba a sobrevolar siempre en los recelos mutuos, pues mientras España consideraría que había un Protectorado doble, que exigía la puesta en común de las decisiones importantes, Francia iba a mirar con desdén lo que consideraba una especie de "subcontrata" otorgada a los generalmente despreciados militares españoles. Por el contrario, la ciudad de Tánger y su territorio (el Fahs) quedaba al margen, como "ciudad internacional”, un estatus que mantendría a lo largo del tiempo (con la excepción de 1940-1945 en que fue ocupada por España). Aún quedarían otros terrenos, los españoles del "Protectorado Sur" en Tarfaya y Cabo Juby, pero por sus condiciones deben considerarse para el caso parte del Sahara Español.

Las premisas administrativas de la administración conjunta hispano-marroquí habrían de sentar las bases de la naturaleza jurídica de los territorios sujetos al protectorado ejercido por España en el Norte de Marruecos, así como, por extensión, de las relaciones entre España y Marruecos como sujetos (activo y pasivo, respectivamente) de dicho protectorado. El sistema administrativo y legal del protectorado hispano-marroquí sentaba sus bases en el respeto integral de la personalidad jurídica internacional de Marruecos así como en la plena soberanía marroquí sobre el territorio protegido (de las dos Zonas, la francesa y la española). El régimen establecido entre ambas naciones mostraba, pues, la dualidad de la naturaleza jurídica del status quo: dos Estados soberanos, con dos administraciones que funcionaban de forma complementaria. De una parte, una administración marroquí con el Jalifa al frente, máxima autoridad marroquí (miembro de la familia del rey, con tratamiento de "alteza imperial") que ejercía sus poderes como representante del Sultán (y en especial la facultad legislativa), expresándolos mediante decretos (dahires). Al mismo tiempo se encontraba al servicio del Jalifa su propio a parato gubernamental, el Majzen, conformado por diversos departamentos (sic) todo bajo la coordinación de un Visir; igualmente existía un consejo consultivo formado por representantes de los territorios de la Zona, y que era designado por el Sultán contando con la propuesta que en este sentido hicieran las autoridades españolas (Verdugo y Parodi, 2010).

En suma, y entrando en materia, a partir de 1912 y hasta 1956 (independencia de Marruecos), deben consignarse tres realidades en la arqueología. Las mismas van a ser absolutamente independientes, y en muy buen parte estancas. Nunca existirán, ni por aproximación, planes de organización, no digamos de investigación, que fueran comunes. Los arqueólogos, o practicantes de la arqueología, desarrollarán sus actividades sin apenas contacto alguno, y con influencias casi nulas. En la zona francesa de Marruecos el gran momento de la arqueología será desde 1916 hasta 1940, por el contrario, en la zona española el papel de la arqueología será mediocre (en calidad) hasta esa última fecha, comenzará a tener cierta relevancia inmediatamente después, y será estelar entre 1949 y 1956. Finalmente, en Tánger nunca existirá un desarrollo arqueológico propiamente dicho, y de hecho, en la última década del Protectorado, los estudios arqueológicos estarán en manos de la Sociedad de Historia y Arqueología de Tánger, organización privada (aunque con apoyo de la administración internacional).

En teoría no debía haber sido así. Las dos potencias coloniales debían asesorar y ayudar a una organización moderna en el país. De hecho, en principio la legislación que se aprobó para la salvaguarda del Patrimonio fue prácticamente común. Y también es una excepción irónica que fuera la administración española la que se adelantara a la francesa en la aprobación del primer texto legal al respecto (en la zona francesa se aprobó el 13 de febrero de 1914).

En la zona española, el Dahir (Orden Real) de 15 de Ramadán de 1331 (es decir, 18 de agosto de 1913), relativo a la conservación de los monumentos y objetos artísticos e históricos (publicado en el Boletín Oficial de la Zona de Protectorado Español, $\mathrm{n}^{\mathrm{o}}$ 13, de 1913), auguraba todas las buenas intenciones para la preservación del legado dejado por nuestros mayores. 
El artículo $1^{0}$ se dirigía a los bienes patrimoniales inmuebles: quedan bajo la salvaguardia del Majzen, que atenderá a su conservación, las construcciones anteriores al Islam, las de los palacios de nuestros antepasados cherifianos, los monumentos religiosos, mezquitas, kubbas, medarsas, etc., las ruinas de las viejas ciudades del Imperio, las fortalezas y las murallas, y en general todas cuantas edificaciones tengan carácter histórico o artístico.

El artículo $2^{\circ}$ indicaba que el Majzen podría ejecutar por su cuenta todos los trabajos necesarios para la conservación de monumentos. Y en el artículo $3^{\circ}$ se incluían los bienes del patrimonio mueble: quedan también bajo la salvaguardia del Majzen todo objeto de arte o antigüedad, como por ejemplo: estatuas, ánforas, columnas, piezas de cerámica, mosaicos, maderas labradas, pinturas, inscripciones históricas y, en general, cuantos tengan un carácter artístico o histórico, quedando prohibida su destrucción, traslado de lugar y su venta con destino al Extranjero, salvo autorización especial. Por todo ello, se encargaba a las autoridades la puesta en ejecución de esta real orden (Valderrama, 1956, 734-735).

En cualquier caso, la implantación de esta legislación de protección del Patrimonio, en Tetuán y su zona en el año 1913, en la zona francesa y más amplia de Marruecos en 1914, significa una novedad y un avance que no pueden menos que ser destacados. Al Magreb más cercano, en el trabajo "civilizador" se llevaba la normativa más moderna. Por hacer legislación comparada, en Alemania hasta el año 1904 no se había creado el instrumento (Heitmatschutz) encargado a nivel estatal de la protección, en Francia la ley de protección del Patrimonio tan sólo se hizo efectiva en ese mismo año de 1913 (Ballart, 1997, 54-55). Y en España, esa misma legislación se encontraba vigente en una primera ley inmediatamente anterior, la Ley de Excavaciones arqueológicas de 1911, a la que seguiría en 1915 la primera Ley más completa, la de Monumentos Históricos y Artísticos (Díaz-Andreu, 2002).

En este punto acabó el valor innovador de la administración española. El Alto Comisario francés, el General Liautey, desde el principio fue plenamente consciente del valor de las antigüedades, y de la exposición del Patrimonio, como justificante de la actuación protectora en la que creía firmemente. Por esta razón le dio prioridad a la investigación en el mundo clásico en la Zona Francesa del Protecto- rado. De este modo, desde el mes de mayo de 1915 tuvo presencia en Marruecos un miembro de la Comission de l'Afrique du Nord, Louis Chatelain. Los franceses crearon el Service des Antiquités, Beaux-Arts et Monuments Historiques, y en el mismo Chatelain estuvo al frente hasta 1941.

Inicialmente el Servicio se instaló en Volubilis en unas condiciones precarias, en las que carecía incluso de tendido eléctrico. En todo caso, la marcha de los trabajos de Louis Chatelain comezó a ofrecer resultados espectaculares, en especial en la propia ciudad de Volubilis, en la limpieza y presentación de las ruinas fueron utilizados los prisioneros alemanes de la Primera Guerra Mundial, y después de agosto de 1916 por parte de trabajadores beréberes. La reconstrucción del arco del triunfo, la restauración del trazado de las calles, vino acompañada del hallazgo de una colección importante de obras de arte, en especial de estatuas, algunos mosaicos, y muchísimas inscripciones latinas. En la exposición colonial de Casablanca, con orgullo de la administración, las antigüedades pudieron mostrarse como muestra de las ventajas del Protectorado, y Chatelain desarrollo una cantidad importante de publicaciones: 4 en 1915, 6 en 1916, 2 en 1917, 6 en 1918, 5 en 1919, 7 en 1920, 10 en 1921, 2 en 1922, etc., (Brouquier-Reddé y Lenoir, 2000).

En los años siguientes continuarían los trabajos de Chatelain, extendidos a Sala (Rabat) y Banasa principalmente. En 1935 el Servicio de Antigüedades llegaba de una forma definitiva a su mayoría de edad mediante la creación de una revista propia, la serie de Publications du Service des Antiquités du Maroc. En cualquier caso, pese a la importancia de sus trabajos, Chatelain en sus estudios se mostró principalmente como un estudioso de las Bellas Artes, en la tradición francesa al respecto (esculturas, mosaicos, construcciones), y como un reputado epigrafista, lo cual quedará bien de manifiesto en sus trabajos, y en sus monografías definitivas (Chatelain, 1942; 1944). Así pues, los trabajos de esta época tendrán también fuertes limitaciones (cerámicas, monedas, distinción de niveles, hipótesis de trabajo), y en el terreno de la interpretación histórica quedarán en manos de Carcopino (1943).

Así pues, pese a lo espectacular de las novedades, lo cierto es que la arqueología francesa en Marruecos hasta las vísperas de la Segunda Guerra Mundial se benefició de la experiencia previa en Túnez y Argelia, con la escuela de Gsell, pero tuvo resultados mucho más limitados. Aún y así, los 
mismos eran absolutamente inalcanzables para los españoles, que carecían de esa experiencia, y sobre todo, de un personal universitario suficientemente formado. Después del Dahir de 1913 la administración española se centró en una labor importante, la organización de la enseñanza, en la que ocupó sus limitados impulsos. Esa labor regeneradora fue destacable, con resultados apreciables, y a ella dedicó lo más granado de la intelectualidad oficial española, pero agotó las fuerzas del débil aparato cultural español.

La única actividad en esta época consistió en parar en Lixus la actuación devastadora de una empresa alemana, que para obtener piedra y emplearla en la construcción del puerto de Larache, utilizaba como cantera la zona de necrópolis Norte de la época romana. Por otra parte, la "limpieza" o desbroce del impracticable campo de ruinas que hacía imposible su visualización y dificultaba la visita, tuvo unos resultados bastante agresivos, como denunció con notable exageración Martinière en una estancia en el lugar: je me suis représenté ce qui serait passé si Lixus avait eu la fortune d'être compris dans le territoire de notre protectorat, donde consideraba que existía una administration éclairée que desarrollaba juiciosos trabajos científicos (Martinière, 1919, 324). Debe tenerse en cuenta que el autor pertenecía desde antiguo al sector que se oponía a que España pudiera tener presencia en Marruecos.

Pasaban los años, y mientras los franceses presumían de labor arqueológica, los españoles no podían enseñar nada. En abril de 1919 se creó la Junta Superior de Monumentos Históricos y Artísticos de Marruecos (JSMHAM), siguiendo el modelo de la metrópoli, que era el organismo encargado de velar por el cumplimiento del Dahir de 1913 (iseis años antes!). Mientras la administración había emprendido la restauración de mezquitas, que iba por su cuenta como edificios religiosos marroquíes en uso, y de algunas zonas de murallas, en concreto en Arcila y en Tetuán. En 1921 la JSMHA de Marruecos realizó la primera iniciativa de carácter arqueológico: encargo a un explorador, César Luis de Montalbán y Mazas, la exploración arqueológica del valle de Tetuán.

Por mucho que Montalbán hubiera visitado Troya, Micenas o el Machu Picchu, en sus recorridos por el Mundo desde 1902, y que su amigo Roso de Luna lo hubiera aficionado a la "arqueología", era totalmente lego en ciencia arqueológica. Aún y así logró reconocer algunos restos importantes, y sobre todo localizó la ciudad antigua de Tamuda, en la que desarrolló excavaciones en 1921 y 1922. El ambiente en el que se desarrollaron las excavaciones es narrado por un viajero, que estuvo un par de semanas en el campamento (Cabrera, 1924), y refleja la inseguridad del momento, época del desastre de Annual, y un Tetuán batido por el cañón de los rifeños, solo salvado por la defensa de la posición de Kudia Tahar, a escasos kilómetros de las ruinas, todo ello aderezado con los relatos continuos y apasionados de Montalbán sobre sus estancias en diversos lugares de todo el mundo.

Montalbán escribía de sus excavaciones unas Memorias, que distaban en todo de ser lo que entendemos por una Memoria Arqueológica, siempre repletas de detalles, anécdotas y digresiones que remotamente vienen al caso. Pero no las publicó porque, en realidad, no eran presentables. Si conocemos la realidad de los primeros hallazgos realizados es sobre todo por el doctísimo informe que realizó en una visita Manuel Gómez Moreno (1922), y de forma subsidiaria, por algunos detalles recogidos por un erudito portugués poco tiempo más tarde (Fomtes, 1924; Gozalbes, 2009). En el año 1923 Montalbán inició las excavaciones en Lixus, que en años sucesivos dirigió en la zona del Foro romano (después identificado por Ponsich como "zona de templos), en las necrópolis, y sobre todo, en el formidable conjunto industrial de las fábricas de salazón de pescado de época romana que, por cierto, durante la mayor parte del tiempo identificó como almacenes del puerto.

Montalbán fue primero nombrado Asesor de la Junta, en 1922, y a partir del año 1926 "Inspector de Excavaciones”. En 1927 se benefició de la prospección de vestigios paleolíticos realizada por Hugo Obermaier (que identificó una quincena de estaciones de superficie con materiales desde el Paleolítico Inferior al Epipaleolítico), de quien aprendió algo de tipología de sílex y cuarcitas, y en esos años realizó otras exploraciones. La JSMHAM estableció una primera exposición permanente, a la que tuvo tendencia de llamar Museo Arqueológico de Tetuán (avant la lettre), primero con los restos de Tamuda. En realidad el Museo Arqueológico de Tetuán, en un local propio, fue inaugurado en noviembre de 1931, ya bajo la Segunda República, y cuando de forma definitiva se recogieron en el mismo los materiales de Lixus. No puede extrañar en la medida en la que el Museo Arqueológico de Rabat no se 
creó hasta esa misma época (1931-1932), por traslado del Service des Antiquités desde Volúbilis.

En lo que respecta a la arqueología prehistórica, en principio la misma estuvo al margen del interés de las autoridades, por cuanto presentaba un menor interés colonial en la propaganda francesa y subsidiariamente española. Existieron no obstante algunos pioneros, como Louis Siret (que aprovechó alguna estancia como ingeniero de minas para realizar prospecciones), o el referido Obermaier en actividad puntual en la zona de Larache-Arcila y Tetuán. Pero sobre todo debe tenerse en cuenta la actuación de dos aficionados muy entregados a la labor, el sacerdote castrense Henry Koehler (que también publicó muchos trabajos sobre el cristianismo en Marruecos), y el entomólogo Maurice Antoine.

Ambos participaron junto a otros aficionados en la creación de la Société de Préhistoire du Maroc (1926), que comenzó a publicar un Bulletin en el que se darían a conocer infinidad de estaciones de superficie. Pese a todo, como diría en broma Antoine, en esas fechas había en Marruecos una Sociedad de Prehistoria, pero sin la sombra de un prehistoriador. Esta primera época de la Société de Préhistoire du Maroc entraría en el descrédito científico, salvado sólo después de la Segunda Guerra Mundial, por la asunción de muchos de sus integrantes de las creencias en la existencia de los Atlantes (que por cierto, Montalbán también compartía).

En todo caso, Antoine prospectó determinadas regiones de Marruecos, con el hallazgo de decenas de estaciones de superficie prehistóricas, teniendo especial potencia los estudios realizados en la Chaouia (región de Casablanca). El P. Koehler también prospectó en superficie, identificó industrias en unos momentos en los que se estaba produciendo su sistematización definitiva en el Magreb (no se produjo hasta los años treinta), y actuó de forma importante al respecto en el territorio de Rabat, y en el de Tánger a Larache (que no publicó, pero donde localizó 18 estaciones). Lo más importante de su actuación fue, sin duda, el estudio de algunas tumbas de la prehistoria reciente en la zona de Tánger, y sobre todo, la excavación en una de las grutas de Achacar, que le permitieron por vez primera identificar la existencia del Neolítico Antiguo (cerámica cardial).

No obstante, la importancia de los hallazgos en Argelia, las dimensiones que la Prehistoria más antigua estaba tomando en el África central de coloni- zación inglesa, relanzó el reconocimiento de la Prehistoria, puesto que la misma comenzaba primero a abrirse un hueco, y luego a ocupar una posición estelar en las disciplinas colonialistas (Robertshaw, 1990). El cambio fue drástico y la administración francesa del Protectorado decidió reconocer y dar estatus oficial a la prehistoria, con lo que precedía los grandes descubrimientos de Cuaternario magrebí.

Fue en 1932 cuando se produjo en Marruecos ese reconocimiento con el nombramiento de Armand Ruhlmann como inspecteur des antiquités préhistoriques del Service des Antiquités. En realidad, cuando llegó a Marruecos, en plena juventud, Ruhlmann sólo había trabajado en el mundo protohistórico y en el de la época romana (Antoine, 1951, 89-90). Desde sus primeros trabajos en Marruecos lograría el reconocimiento como especialista en Prehistoria (recién creado en Francia), y la primera tesis doctoral sobre prehistoria marroquí con la investigación sobre las grutas de El Khenzira en la zona de Mazagán, publicada en 1936.

Su trabajo era el característico de la época, por lo que aportó como novedad fundamental el trabajar siempre con geólogos (más adelante lo haría con René Neuville, llegado en 1940 del destino consular en Gibraltar), con lo que superaba la etapa de sus contemporáneos aficionados: hizo prospecciones en las que localizó cantidad de estaciones de superficie, sistematizó por vez primera el Paleolítico y Epipaleolítico de Marruecos, excavó en las grutas de El Khenzira y en Dar es Soltane, y terminaría de forma trágica muriendo en accidente en acto de servicio, en la excavación de la cueva de El Aouïn en el Marruecos oriental (1948).

Por la parte española, nada hay que pueda compararse en relación con la prehistoria. En cualquier caso, en la época de la Segunda República, después de la inauguración del Museo Arqueológico de Tetuán, y de la publicación del primitivo Mapa arqueológico de Montalbán (1933), se diseña un proyecto estelar que va ser la excavación en el monumento protohistórico de M'Zora (Chouahed, en la zona de Arcila). Se trata de una actividad que cierra la etapa anterior (en resultados), pero abre la siguiente (en lo que respecta a objetivos). Se trataba de explorar y hacer visitable el monumento con vistas a su conversión en una especie de "parque arqueológico”, para hacerlo centro de atracción del turismo. Sus condiciones eran innegables: un círculo de monolitos de unos 54 metros de diámetro, con 
un corredor interior enlosado, y con un túmulo de tierra en su interior que llegaba hasta los 6 metros de altura.

Los trabajos se inician en 1932-1933 con la limpieza, que dio resultados positivos, pues dejó al descubierto las características del monumento, con su número exacto de monolitos, y la existencia del enlosado, y del muro de contención de la tierra del túmulo (con aparejo de tipo helenístico). Pero los problemas se acumularon a partir del proceso de excavación en el túmulo artificial, fuertemente afectado por resultados inesperados (no era un sepulcro de corredor como se esperaba) y por la impericia de Montalbán. Para rematar la desgracia, perdiéndose los datos (hallazgo de una cista con algún objeto metálico en forma de puñal o pequeña espada, a partir de diversos testimonios orales), en julio de 1936 Montalbán fue detenido, a punta de fusil, por las tropas franquistas, produciéndose después el saqueo y destrucción de lo descubierto, y el deterioro de las paredes, todo ello en la búsqueda de tesoros.

El periodo posterior en la arqueología marroquí se caracteriza por la coincidencia con las guerras. Primero con la guerra civil española que, obviamente, imposibilita los estudios por parte de los españoles. Después por la Guerra Mundial, que obviamente afectará a Francia, en la evolución de su situación, desde la guerra con Alemania, el régimen de Vichy (en el que Carcopino fue ministro), el paso a la Francia libre y a los Aliados. También en Tánger se producen novedades puesto que por vez primera desde la época inicial del siglo se van a producir estudios, y la ciudad, como veremos, atraerá la atención de los investigadores, pero se verá afectada por la ocupación española en 1940.

Por la parte francesa esta nueva etapa se iniciará con la ya referida creación de la revista especializada: Publications du Service des Antiquités du Maroc. Desde el primer número vemos la presencia de los pesos pesados de la arqueología marroquí (Chatelain y Rulhmann, pero con la aparición de una nueva figura: Raymond Thouvenot. Después de sus trabajos en Roma, Thouvenot había sido miembro del Institut des Hautes Études Hispaniques (posterior Casa de Velázquez; a todo lo largo de los años treinta había estado presente en España, donde consiguió su especialización, y en 1936 tenía ya terminada su tesis doctoral, que publicaría cuatro años más tarde, dedicada a una síntesis sobre la Bética romana, convertida en emblemática en la bibliografía.
Thouvenot ya había tenido presencia y había realizado trabajos en Marruecos, cuando desde época de la guerra civil española llegó destinado como adjunto de Chatelain, a quien sustituiría en 1941. Sus excavaciones inicialmente se centrarían en $B a$ nasa, colonia romana creada por Augusto, acerca de la que publicaría pronto una síntesis (Thouvenot, 1941). El volumen de publicaciones de Thouvenot sobre Marruecos era muy similar al del citado Chatelain, incluso en las circunstancias de la guerra: 9 en 1935, 3 en 1936, 2 en 1937, 4 en 1938, 5 en 1939, 2 en 1940, 7 en 1941, 2 en 1942, 0 en 1943, 2 en 1944, 10 en 1945 (Brouquier-Reddé y Lenoir, 2000).

Pero lo más importante es que el investigador ya representaba una nueva generación respecto a Chatelain, y su trabajo mostraba que el arqueólogo debía rebasar el terreno de las Bellas Artes, o la mera especialización epigráfica. De esta forma, Thouvenot va a mostrar en sus múltiples publicaciones un dominio bastante mayor de las fuentes literarias, algunos trabajos serán específicos al respecto, y sobre todo también del uso y estudio de las monedas, en parte de la cerámica siempre olvidada por Chatelain, o de las ánforas. Así pues, Thouvenot fue un arqueólogo mucho más completo que Chatelain, porque su formación ya había sido más específica, y porque el concepto de arqueología ya había cambiado.

En cualquier caso, si es cierto que Thouvenot era un historiador que consideraba la arqueología una magnífica fuente de documentación, desde la perspectiva de la interpretación histórica, representó una cierta regresión colonialista en relación con Chatelain. Éste, al igual que Carcopino, observaban la antigua Tingitana romana con un valor por sí misma, con unas transformaciones importantes de las sociedades indígenas que, en una buena parte, habían colaborado y auspiciado la conquista romana y la romanización. Se trataba de una visión optimista sobre las propias potencialidades de la colonización francesa, que después del enfrentamiento armado no tendría resistencias hasta los años treinta.

Por el contrario, Thouvenot procedía del estudio de "su" querida Bética, y ante ella, la Tingitana no podía tener sino el contraste de un territorio bárbaro. Así Thouvenot dio un par de vueltas de tuercas a la imagen de militarización de la Tingitana, con base en estudios suyos como las incursiones de moros en la Bética, o Roma y los "bárbaros" africanos. De esta forma, para Thouvenot, más allá 
de algunas producciones culturales brillantes, no era sino un guardaespaldas avanzado de la Bética, único justificante de ocupación para evitar las incursiones de los moros. Una imagen que tan sólo Miguel Tarradell, años más tarde, lograría superar al incidir en los componentes económicos (agricultura, salazones de pescado), que Michel Ponsich en los años sesenta terminaría por remachar.

Estos años anteriores a la ocupación española de 1940, en la Guerra Mundial, la arqueología de Tánger había cobrado cierto protagonismo. La colección arqueológica existente desde la época de la Mision Scientifique en el año 1928 se convirtió de forma definitiva en Museo de la Kasba. El Museo siguió dependiendo del Consulado francés, dependiente del Servicio de Enseñanza Francesa en Tánger. En los años treinta aparecieron algunas estatuas romanas, en especial la de la mujer con túnica aparecida en 1935 en el Zoco Grande, y que motivaron una estancia de Chatelain, que en esos años publicó algunos trabajos sobre estas obras.

En Tánger confluyeron dos aficionados norteamericanos, el médico Ralph Nahon, que desde al menos 1936 recorría el territorio tangerino, y poseía una colección de piezas de sílex, y Hooker A. Doolitle, encargado de negocios de los EE.UU. Estos dos personajes en 1938 y 1939 realizaron algunas excavaciones por su cuenta. De ellas tenemos en general muy pocas noticias, circunscritas a que en esos años excavaron en la cueva grande o alta de las grutas de Hércules, de nombre indígena Mugharet el Aliya, gruta que años más tarde (a partir de 1947) alcanzaría gran fama por los descubrimientos realizados en la misma.

En 1939 se sumó a las excavaciones, ya más científicas, el profesor Coon, que a la profundidad de 6 metros extrajo restos fósiles humanos; en 1940 Nahon y Doolittle continuaron las excavaciones, conectados por radio con las instrucciones de Coon, y en 1941 los restos salieron para el Peabody $\mathrm{Mu}$ seum de la Universidad de Harvard. Las excavaciones se retomarían, en plan ya más científico, a partir de 1947 y ofrecerían ciertamente resultados muy importantes para el Paleolítico y Neolítico. Las peculiares circunstancias y cautela con la que deben tomarse los resultados, han sido expuestas por uno de nosotros en otra ocasión (Gozalbes, 2007), pero en todo caso al entrar en la ciudad los españoles en 1940 clausuraron las búsquedas e instalaron una batería antiaérea en el lugar.
La CONSOLIDACión DE LAS ESTRUCTURAS (19391946)

El período contemporáneo al desarrollo de la II Guerra Mundial estaría marcado, en lo relativo a la arqueología en la Zona Española del Protectorado, por las figuras de Pelayo Quintero Atauri (nacido en Uclés, provincia de Cuenca, en 1867 , y fallecido en Tetuán, en 1946) y de Tomás García Figueras (Jerez de la Frontera, 1892-1981), quien, desde sus diversos cargos de responsabilidad en el ámbito de la Alta Comisaría Española (García Figueras, militar de formación, ejercería, entre otras funciones, como delegado de Economía, de Educación y Cultura y de Asuntos Indígenas, o como Secretario General de la Alta Comisaría, permaneciendo en puestos de responsabilidad de forma ininterrumpida desde los años de la Guerra Civil hasta la desaparición del Protectorado, en los años 50, siendo una de las figuras clave en la administración española del Norte de Marruecos).

Sería Tomás García Figueras quien diseñase y llevase a la práctica la puesta en funcionamiento de las estructuras de gestión cultural en el seno de la administración española del Norte de Marruecos desde 1936, fruto de lo cual sería la rehabilitación de Montalbán, la reintegración en el servicio de Bellas Artes de Mariano Bertuchi (como responsable del mismo), la creación del Servicio de Antigüedades (de Arqueología) o la puesta en marcha del nuevo Museo Arqueológico, instituciones al frente de las cuales García Figueras -no sin contar con la complicidad y ayuda del entonces Alto Comisario, Juan Beigbeder- habría de situar a Pelayo Quintero de Atauri. Nacido en los estertores del reinado de Isabel II y formado en la España de la Restauración, sobrino del histórico excavador de Segóbriga Román García Soria, alineado políticamente con los liberales de Sagasta y Moret, y miembro, luego, de la Unión patriótica de Primo de Rivera, reunía un perfil poliédrico en el que se unían sus facetas de historiador, arqueólogo, profesor, director de $\mathrm{Mu}-$ seos, gestor de Patrimonio, y los no pocos cargos -todos, a excepción de su puesto como profesor de la Escuela de Bellas Artes de Cádiz, no remunerados, que fue acumulando a lo largo de su vida.

No nos extenderemos en el perfil profesional y personal de Quintero, por haberlo abordado en ocasiones anteriores (Parodi, 2007; 2008; 2008b; 2008c; Parodi y Gozalbes, 2011; Verdugo y Parodi, 2010; Zouak y Parodi, 2011). Señalaremos que, alejado ideológicamente del golpe de estado de julio 
de 1936, este arqueólogo y gestor público acabaría sus días en Tetuán falleciendo en 1946, debiendo abandonar la provincia de Cádiz (donde había desempeñado buena parte de sus responsabilidades desde los primeros años del siglo XX y hasta 1939) por instigación de determinados personajes afectos al régimen franquista y siendo acogido (a sus más de 70 años, en 1939) en las estructuras de gestión del Patrimonio Cultural del Norte de Marruecos bajo coadministración hispano-marroquí, contando como sus valedores a los ya mencionados Juan Beigbeder Atienza y Tomás García Figueras, verdadero "hombre fuerte" de la administración española en el septentrión marroquí.

El trabajo de campo del Servicio de Excavaciones de la Alta Comisaría Española contando con Pelayo Quintero al frente como responsable, y especialmente el trabajo del propio Quintero como arqueólogo (con un Montalbán rehabilitado y reincorporado al trabajo, al frente del sector occidental del territorio, con sede en la ciudad de Larache y bajo la autoridad directa de Quintero y de García Figueras), habría de centrarse casi exclusivamente en el yacimiento arqueológico de Tamuda, resultante de una unión de factores entre los que se contarían la escasa capacidad presupuestaria del momento (una constante en la realidad de la arqueología de la época, de acuerdo con los testimonios a este respecto del propio Quintero ya antes de su llegada a Marruecos, en ámbito gaditano), la proximidad de este sitio arqueológico a la ciudad de Tetuán y la existencia de trabajos previos (los desarrollados antes de la guerra civil por Montalbán), que podrían hacer más atractiva la continuación de las labores de investigación en este yacimiento, así como (ya en un plano humano) la propias circunstancias vitales de un Pelayo Quintero anciano (que emprende su primara campaña de campo en el verano marroquí de 1940, a la edad de 73 años), enfermo y progresivamente agotado. Se emprendieron entonces (entre 1940 y 1945, en plena Segunda Guerra Mundial -que no afectó directamente al territorio de la Zona Española, pero que es la "envolvente general”, por así decirlo, en que se enmarcan los trabajos y la realidad del momento) seis campañas de investigación arqueológica en el yacimiento de Tamuda, estando las de los años 1940 y 1941 bajo la dirección de Quintero Atauri, y las de 1942, 1943, 1944 y 1945 bajo la dirección conjunta de Quintero y su secretario en el Museo, Giménez Bernal; estos trabajos sirvieron para ampliar la zona ya excavada en dicho yacimiento por César Luis de Montalbán, al tiempo que se enriquecían los fondos del Museo Arqueológico tetuaní con los materiales procedentes de estas excavaciones.

Además de las mencionadas campañas arqueológicas llevadas a cabo por Quintero Atauri entre los años 1940 y 1945 (que cerrarían cronológicamente este capítulo) es conveniente hacer mención igualmente de la del año 1946, que se desarrollaría ya definitivamente postrado Quintero (precisamente poco tiempo antes de su fallecimiento, en octubre de dicho año), y que fuera dirigida por el sacerdote agustino César Morán junto al referido Cecilio Giménez. César Morán Bardón, antropólogo, etnólogo, estudioso de las lenguas y tradiciones populares castellanas y arqueólogo aficionado, sería la persona en quien Quintero habría depositado su confianza para sucederle en las responsabilidades arqueológicas en el Norte de Marruecos, aunque otras circunstancias -fundamentalmente los conflictos de intereses y las luchas de poder en el seno de la Arqueología española de la época, entre la Comisaría General de Martínez Santaolalla y sus adversarios del campo universitario, que se sumarían a las debilidades académicas y de salud de Morán) resolverían la situación a favor de Miguel Tarradell (Díaz-Andreu, 2002; Gozalbes y Parodi, 2011).

Quintero centraría sus esfuerzos en los sectores meridionales y occidentales del yacimiento en su facies romana, en las murallas, los torreones, la puerta y la zona habitacional interior del espacio interior del castrum, aunando los trabajos de campo con la publicación anual de las correspondientes "Memorias" de los mismos en las que aparecen publicados los resultados de sus investigaciones. Los estudios de campo tenían así un vehículo de expresión y un paralelo en la publicación y divulgación de los mismos (una constante vital en la rutina de trabajo de Quintero sería precisamente la de publicar lo que investigaba, no dejando nada al olvido), algo que el de Uclés llevaría a cabo mediante el empleo de todos los recursos a su alcance (en una época de especial penuria económica), tales como los medios de prensa existentes en el ámbito de la Zona Española del Protectorado, revistas divulgativas como Mauritania (editada por los franciscanos), o las Memorias de los Museos provinciales, en las que el Museo de Tetuán no estaba inicialmente incluido y que desde 1943 albergarían la Memoria anual del tetuaní por iniciativa de Quintero, con lo que se insertaba a esta institución del Patrimonio y centro 
de investigación en un circuito mucho más amplio, dado que se entraba en una dinámica general, de ámbito estatal español, lo que permitiría una mayor difusión de las investigaciones desarrolladas en el norte de África entre estudiosos nacionales y extranjeros, reflejo de lo cual, quizá, serían las visitas de estudios a Tetuán como las llevadas a cabo por la entonces directora del Museo de Granada, una joven Josefina Eguarás).

Los trabajos de investigación efectuados por César L. de Montalbán desde principios de los años 20 del siglo pasado (en yacimientos como los de Lixus, Mezora o Tamuda) hacían necesario disponer de un Museo para albergar los materiales fruto de dichos trabajos (hasta entonces almacenados en instalaciones de Larache y Tetuán). Así, ya en la década de los 20 del siglo XX se habría contado con depósitos o almacenes que habrían servido a estos fines. Finalmente, y como consecuencia de varios factores entre los que cabe contar la propia y paulatina puesta en funcionamiento de las estructuras administrativas y de gestión del Patrimonio Histórico en el Protectorado, así como del desarrollo de los trabajos de campo (igualmente ligados al desarrollo de las referidas estructuras) que inicialmente echaron a andar a principios de los años 20 del siglo pasado, y ampliamente superadas las posibilidades de los depósitos y almacenes existentes, en la ciudad de Tetuán se habilitaron unas dependencias de un edificio de la calle Mohammed Torres (en el no․ 7), en las que habría quedado establecido el Museo en el mes de noviembre de 1931; el responsable de dicho Museo debió ser César L. de Montalbán.

Andando el tiempo, pocos años más tarde, en 1938 (mismo año de la inauguración de otros dos señeros equipamientos culturales de Tetuán: la Biblioteca General y la Hemeroteca), se decidiría trasladar el Museo (cuyas instalaciones eran ya insuficientes, lo que lleva a pensar que lo fueran ya desde inicio), comenzando las obras de la nueva sede en 1939, en la calle Mohammed ben Hossain, junto al Bajalato de la ciudad, justo fuera de la Medina y cerca de una de sus Puertas; el nuevo Museo se situaba (y se sitúa) en una zona axial, de confluencia entre las dos áreas urbanas bien identificadas de la Tetuán histórica: la Medina medieval y el Ensanche español, y se convertiría en el vehículo articulador de la gestión del Patrimonio Arqueológico en el territorio del Norte de Marruecos desde el mismo momento de su puesta en funcionamiento, en el verano de 1940 (el 19 de julio de dicho año, fecha no dejada al azar), merced al impulso de su director, Pelayo Quintero, quien habría de ejercer una doble responsabilidad: inspector de excavaciones y director del Museo Arqueológico de Tetuán; Quintero Atauri vino a ser la figura de máxima autoridad en materia de gestión pública del Patrimonio Arqueológico en el Norte de Marruecos bajo administración conjunta hispano-marroquí, bajo la dirección de las autoridades de la Alta Comisaría Española (donde el ya mencionado Tomás García Figueras jugaría un papel esencial) y del Majzén (la administración marroquí bajo la autoridad del Jalifa) entre 1939 (año de su entrada en Marruecos) y 1946 (año de su fallecimiento en Tetuán).

Este Museo de la calle Ben Hossain habría de convertirse desde su puesta en marcha, como hemos adelantado, en el eje articulador y en el elemento nuclear del trabajo de investigación arqueológica de campo, y funcionaría como elemento rector de la investigación y el trabajo sobre el terreno, gracias, además, a la labor de su director, Quintero, al frente del servicio de la Inspección General de Excavaciones del Protectorado, lo que se conjugaba con sus responsabilidades al frente del propio Museo, una institución del Patrimonio que habría de ser mucho más de un mero "depósito de colecciones" (como habría sido el caso de las instalaciones precedentes, de los años 20 y de 1931), alejándose definitivamente del perfil de "almacén de piezas" (procedentes de las excavaciones de yacimientos arqueológicos del territorio) que sí habrían tenido sus precedentes, y que se convertiría en un núcleo activo de la investigación, la gestión y la difusión del Patrimonio Arqueológico del territorio bajo su responsabilidad.

La figura de Quintero Atauri en el marco de la Arqueología del Norte de Marruecos marcaría el ocaso de una época, la de la Arqueología como una realidad academicista y voluntarista, aún impregnada de anticuarismo, y el principio de otra, la de la Arqueología académica, científica y considerada como una rama de la administración pública, de la gestión del Patrimonio Histórico. Tras un breve lapso (comprendido entre la muerte de Quintero, en 1946 y la llegada de quien habría de sucederle en sus responsabilidades, Miquel Tarradell, en 1948) se abriría paso a una nueva etapa, con la que se alcanzaría la consolidación de una Arqueología "nueva”, profesional, universitaria e imbricada en la gestión pública del Patrimonio en el Norte de Marruecos (de manera pareja y paralela a lo que vendría sucediendo en España). 
Los difíciles años de la II Guerra Mundial habrían de representar en el Norte de Marruecos, en la Zona Española del Protectorado, y en lo relativo a la Arqueología en el territorio bajo gestión conjunta hispano-marroquí, un momento de establecimiento y de construcción de estructuras de gestión del Patrimonio Arqueológico del territorio (si bien es de señalar que esto no sería algo exclusivamente orientado a la materia arqueológica). Mientras el resto del mundo se hacía la guerra, en el Norte de Marruecos, pese a las dificultades propias del momento y al propio cariz de la situación del territorio, se estaban sentando las bases de la gestión del Patrimonio Histórico desde el prisma de una administración a la, que no podemos considerar sino en su contexto histórico, social, económico y político. Quintero, primero, y Tarradell inmediatamente después, sentarían los cimientos ( a García Figueras, "motor remoto" de esta acción) de la conservación, la investigación, la difusión y la aplicación de la protección del Patrimonio Arqueológico del Norte de Marruecos en los años 40 y 50 del siglo pasado.

\section{BiBLIOGRAFÍA}

Antoine, M. (1951), "Le développement des études préhistoriques au Maroc", Bulletin de la Société de Préhistoire du Maroc, 2, 3/4, 85-99.

Ballart, J. (1997), El patrimonio histórico y arqueológico: valor y uso, Barcelona.

Brouquier-Reddé, V. (2000), "Les brigades topographiques au Maroc (plaine du Gharb et région de Volubilis", L'Africa Romana, XIII, 959989.

Brouquier-Reddé, V. y Lenoir, E. (2000), "Bibliographie du Maroc Antique", L'Africa Romana, XIII, 991-1072.

Cabrera, A. (1924), Magreb el Aksa. Recuerdos de cuatro viajes por Yebala y por el Rif, Madrid.

Carcopino, J. (1943), Le Maroc Antique, París.

Chatelain, L. (1942), Inscriptions Latines du Maroc, Rabat.

(1944), Le Maroc des romains, París.

Díaz-Andreu, M. (2002), Historia de la Arqueología. Estudios, Madrid.

Euzennat, M. (1956), "Deux voyageurs anglais à Volúbilis (1721)”, Hespéris, 43, 325-334.

Fontes, J. (1924), "Impressoes duma visita a Tamuda e Tetuao", Arqueologia e Historia, 3, 75-87.

Ghottes, M. y Parodi Álvarez, M.J. (2011), "Le fleu- ve Tamuda", Pelayo Quintero en el primer centenario de 1912 (Parodi, M.J. y Gozalbes, E., Eds.), Cádiz, 423-454.

Gómez Moreno, M.(1922), Descubrimientos y antigüedades en Tetuán, Suplemento del número de 10 de noviembre del Boletín Oficial de la Zona de Protectorado español en Marruecos, Madrid.

Gozalbes Cravioto, E. (2002), "Las excavaciones arqueológicas de 1921-1922 en Tamuda (Tetuán, Marruecos)", Cuadernos del Archivo Central de Ceuta, 14, 325-342.

(2005), "Los pioneros de la Arqueología española en Marruecos (1880-1921)", El nacimiento de la Prehistoria y la Arqueología científica (Cabrera, V. y Ayarzagüena, M., Eds.), Madrid, 110-117.

(2007), “Algunos avatares de la arqueología colonial en el Norte de Marruecos (1939-1942)”, Boletín de la Asociación Española de Orientalistas, 42, 77-95.

(2008a): "Los españoles y las antigüedades de Marruecos: de Ali Bey el Abbasi al inicio del Protectorado (1800-1936)", Historia de la Arqueología en el Norte de Marruecos durante el periodo del Protectorado y sus referentes en España (Beltrán, J. y Habibi, M., Eds.), Sevilla, 63-95.

(2008b): "Los primeros pasos de la arqueología en el Norte de Marruecos”, En la orilla africana del Círculo del Estrecho. Historiografía y proyectos actuales, Colección de Monografías del Museo Arqueológico de Tetuán II (Bernal, D., Raissouni, B., Ramos, J., Zouak, M. y Parodi, M.J., Eds.), Cádiz, 33-61.

(2008c), "La Arqueología española en Marruecos (1921-1936). Memorias y desmemorias”, Documentos inéditos para la Historia de la Arqueología (Mora, G. y Ayarzagüena, M., Eds.), Madrid, 183-195.

(2008d), "Notas de historiografía arqueológica: la visita de Joaquim Fontes a Tetuán y Tamuda (Marruecos) en 1923”, Revista Portuguesa de Arqueologia, 11 /1, 285-295.

Gozalbes Cravioto, E. y Parodi Álvarez, M.J. (2011), "Miguel Tarradell y la Arqueología del Norte de Marruecos”, Actas del III Seminario Hispano Marroquí. Arqueología y Turismo, Colección de Monografías del Museo Arqueológico de Tetuán III (AA.VV.), Cádiz, 105-127. 
La Martinière, H. (1919), Souvenirs du Maroc. Voyages et missions, París.

Montalbán, C.L. (1933), Mapa arqueológico de la Zona de Protectorado Español en Marruecos, Madrid.

Parodi Álvarez, M.J. (2006), "La Razón de la Sinrazón. Cayo César, el obelisco y las lentejas”, Economía de prestigio versus Economía de mercado (G. Chic García, Ed.), Sevilla, 89-101.

(2007), “Arqueología española en Marruecos, 19391946. Pelayo Quintero de Atauri”, Spal, 15, 9-20.

(2008), "Notas sobre Historiografía Arqueológica Hispano-Marroquí. 1939-1946, Pelayo Quintero", En la orilla africana del Círculo del Estrecho. Historiografía y proyectos actuales, Colección de Monografías del Museo Arqueológico de Tetuán II (Bernal, D., Raissouni, B., Ramos, J., Zouak, M. y Parodi, M.J., Eds.), Cádiz, 63-92.

(2008b), "Pelayo Quintero: Arqueología en las dos orillas del Fretum Gaditanum", L'Africa Romana, XVII, 2517-2526.

(2008c): "Pelayo Quintero de Atauri. Apuntes de Arqueología hispano-marroquí, 1939-1946”, Historia de la Arqueología en el Norte de Marruecos durante el Protectorado y sus referentes en España (Beltrán, J. y Habibi, M., eds.), Sevilla, 97-119.

(2009), "Notas sobre la organización administrativa de las estructuras de gestión del Patrimonio Arqueológico en el Marruecos Septentrional durante el Protectorado (1912-1956)", Herakleion, 2, 117-141.

(2011), "Pelayo Quintero. Crepúsculo en Tetuán”, Pelayo Quintero en el primer centenario de 1912 (Parodi, M.J. y Gozalbes, E., Eds.), Cádiz, 309-322.

(e.p.), "El admirable crepúsculo: Pelayo Quintero y la Arqueología en el Norte de Marruecos”, $A c$ tas de las Jornadas "Pelayo Quintero Atauri. El Sabio de Uclés”, (Gozalbes, E. y Parodi, M.J., Eds.).

Parodi Álvarez, M.J. y Gozalbes Cravioto, E. -Coords. ed.- (2011), Pelayo Quintero en el primer centenario de 1912, Cádiz.

(2011), "La Arqueología en el Norte de Marruecos (1900-1945)”, Actas del III Seminario Hispano Marroquí. Arqueología y Turismo (AA. VV.), Cádiz, 137-159.
Rebuffat, R. (2000), "Histoire de l'identification des sites urbaines antiques du Maroc", L'Africa Romana, XIII, 865-914.

Souville, G. (1973), Atlas préhistorique du Maroc. 1. Le Maroc Atlantique, París.

Thouvenot, R. (1941), Une colonie romaine de Maurétanie Tingitane: Valentia Banasa, París.

Valderrama, F. (1956), Historia de la acción cultural de España en Marruecos (1912-1956), Tetuán.

Verdugo Santos, J. y Parodi Álvarez, M.J. (2010), "La gestión del Patrimonio Arqueológico en el antiguo Protectorado español en el Norte de Marruecos. Gestión, administración, normativas”, Spal, 17, 9-25.

Verdugo, J., et alii (2011), “Tamuda. De la arqueología colonial a la Cooperación Internacional”, Pelayo Quintero en el primer centenario de 1912, (Parodi, M.J. y Gozalbes, E., Eds.), Cádiz, 355-420.

Zouak, M. y Parodi Álvarez, M.J. (2011), "Pelayo Quintero y el Arqueológico de Tetuán”, Pelayo Quintero en el primer centenario de 1912, (Parodi, M.J. y Gozalbes, E., Eds.), Cádiz, 325352. 